\title{
ORIGINAL
}

\section{PERFIL ANTROPOMÉTRICO Y PREVALENCIA DE SOBREPESO DE LOS ESCOLARES DE UBRIQUE, CÁDIZ}

\author{
Inmaculada Failde Martínez, Juan Zafra Mezcua, Jose Pedro Novalbos Ruiz, Manuel Costa \\ Alonso y Enrique Ruiz Rodríguez.
}

Area Medicina Preventiva y Salud Pública. Universidad de Cádiz.

\section{RESUMEN}

Fundamento: La obesidad del adulto es un importante factor de riesgo de diversas enfermedades crónicas, y parece ligada a la ohesidad en la infancia. Por ello identificar a niños con riesgo de obesidad, podría ser una medida adecuada de prevención de estas enfermedades en el adulto

El objetivo de nuestro estudio fue conocer el perfil antropométrico y la prevalencia de sobrepeso de una población escolar de un núcleo rural de Cádiz con un importante carácter industrial.

Método: Se realizó un estudio transversal donde se estudió una muestra representativa de 493 escolares de ambos sexos entre 4 y 14 años de edad, en los que se realizaron mediciones de la talla, peso, perímetro braquial, pliegue cutáneo tricipital, y se calculó el IMC. Los resultados se compararon con una población española de referencia. Como medida de sobrepeso se utilizó el IMC $\geq 25 \mathrm{Kg} / \mathrm{m}^{2}$.

Resultados: La talla, el peso, el perímetro braquial y el pliegue cutáneo tricipital y el IMC. presentaron valores algo superiores a la población de referencia, especialmente en las edades mayores. La Prevalencia de sobrepeso fue de $4.05 \%$, con un mayor número de casos a partir de los 12 años y sin que se apreciaran diferencias por sexos.

Conclusiones: Los escolares presentaron un patrón antropométrico ligeramente superior a la población de referencia, y una baja prevalencia de sobrepeso. Estos resultados están en línea con los publicados por los autores sobre la alimentación de esta población.

Palabras clave: Antropometría. Sobrepeso. Escolares. Pliegue Cutáneo Tricipital. IMC. Perímetro Braquial.

\section{ABSTRACT}

\section{Anthropometric Profile and Prevalence of Overweight among Schoolchildren in Ubrique, Cadiz}

Background: adult overweight is an important risk factor for a range of chronic illnesses, and seems to be linked with overweight in childhood. The identification of children at risk of overweight could therefore be a suitable means of preventing these illnesses in adults. The aim of our study was to determine the anthropometric profile and prevalence of overweight in a population of schoolchildren in a rural town in Cadiz, which is also a major industrial centre.

Method: a cross-sectional study was carried out, in which a representative sample of 493 schoolchildren of both sexes and aged from 4 to 14 years old was studied. They were measured for height, weight, arm circumference, triceps skinfold, and their IMC was calculated. The results were compared with a Spanish reference population. To measure obesity IMC $\geq 25 \mathrm{~kg} / \mathrm{m}^{2}$ was used.

Results: height, weight, arm circumference, triceps skinfold and the IMC showed values that were slightly above those corresponding to the reference population, and there was a low incidence of overweight. These results are along the lines of studies published by the authors on the eating habits of this population.

Key words: Anthropometry. Overweight. Schoolchildren. Triceps Skinfold. IMC. Arm circumference.
Correspondencia

Inmaculada Failde Martínez

E.U. Ciencias de la Salud. Universidad de Cádiz

C/ Duque de Nájera 18. Apdo. Correos 621

11003 Cádiz

Tfno: (956) 211064 Fax (956) 224218

Correo electrónico: inmaculada.failde@uca.es

\section{INTRODUCCIÓN}

El crecimiento y desarrollo son dos procesos continuos que se inician en la concepción y continúan durante la infancia y adolescencia. Estos procesos no son lineales, sino que se caracterizan por presentar aceleraciones y desaceleraciones ${ }^{1}$. En su regula- 
ción van a influir una serie de factores endógenos (genéticos, metabólicos y neurohormonales) y exógenos (nutricionales y medioambientales).

Para que el crecimiento y desarrollo se realicen con normalidad es necesario un aporte suficiente de nutrientes y energía. En los países desarrollados no es frecuente la carencia de calorías o proteínas, siendo mucho más usual el exceso de aporte energético que produce almacenamiento en el tejido adiposo.

La obesidad se ha considerado un importante factor de riesgo para el desarrollo de ciertas enfermedades crónicas como la hipertensión arterial, diabetes, enfermedad coronaria e incluso algunas neoplasias preferentemente en el sexo femenino ${ }^{2}$.

La obesidad del adulto puede iniciarse a cualquier edad, pero existen argumentos a favor de que cuando esta comienza en la niñez o en la adolescencia, tiende a perdurar toda la vida. Diversos autores ${ }^{2,3}$, hablan de que la proporción de niños obesos que se transforman en adultos obesos crece con la edad y con la severidad del cuadro, variando desde el $26-41 \%$ para la edad preescolar hasta el $63 \%$ en la edad escolar y adolescencia. Así mismo se habla de que el riesgo de padecer obesidad en la edad adulta es al menos 2 veces superior para los niños obesos que para los no obesos ${ }^{3,4}$.

La prevalencia de obesidad en la infancia varia entre $1-28 \%$. Este rango tan amplio de valores se debe mayormente a los diferentes criterios en la definición de obesidad y a los distintos valores de referencia utilizados en los estudios. A pesar de esto, los parámetros antropométricos se han usado frecuentemente para definirla y se han considerado esenciales para obtener una información básica de la población especialmente cuando se pretende tener una perspectiva clínica y epidemiológica.

Los parámetros antropométricos de mayor uso son el peso, la talla las circunferen- cias y los pliegues, con los que se establecen diferentes índices como el Índice de Masa Corporal (IMC).

En estudios que miden tanto los pliegues cutáneos como el IMC, la correlación de estos con la obesidad es generalmente más fuerte para el IMC, debido a que el peso y la talla están menos sujetos a errores de medida que los pliegues. Por otra parte, el IMC mide la masa corporal magra y el tejido adiposo, a diferencia de los pliegues que miden fundamentalmente la masa de tejido graso, más sujeta a modificaciones a lo largo del tiempo.

En base a lo anterior nos propusimos realizar un estudio con el objetivo de conocer el perfil antropométrico y la prevalencia de sobrepeso de los escolares de una población rural de la Sierra de Cádiz, con el fin de identificar los niños a riesgo de obesidad sobre los que se podría realizar una intervención temprana encaminada a reducir el sobrepeso y por tanto a prevenir la obesidad en la edad adulta y sus consecuencias sobre la salud.

\section{SUJETOS Y MÉTODO}

Se trata de un estudio transversal, que forma parte de otro trabajo más amplio sobre valoración del estado nutricional ${ }^{6}$, donde la población objeto de estudio estaba formada por los alumnos matriculados en E.G.B en los siete colegios que existen en la localidad de Ubrique (Cádiz), con un total de 3.352 escolares.

Se eligió una muestra aleatoria simple, estratificada por edad (4-14 años), sexo y colegio, a partir de las listas de escolares proporcionadas por cada centro.

Para el cálculo del tamaño de la muestra se utilizaron las tablas para poblaciones finitas y proporciones, considerando un margen de error del $4 \%$ y un intervalo de confianza del $95 \%$. La muestra elegida fue de 493 
alumnos sanos que no tomaban medicación anabolizante alguna.

Para la valoración antropométrica se recogió la información sobre talla, peso, perímetro braquial izquierdo y pliegue cutáneo tricipital izquierdo de todos los escolares seleccionados.

Para medir la talla, se utilizó un tallímetro vertical con una escala graduada en centímetros y con una exactitud de $1 \mathrm{~mm}$. Se tomó la talla en posición erecta, sin zapatos ni calcetines, con el cuerpo estirado y mirando al infinito.

El peso se midió con una báscula de plataforma, de capacidad para 130 kilogramos y con una exactitud de 100 gramos. Los escolares fueron pesados, conservando únicamente su ropa interior. A partir del peso y la talla se calculó el IMC (peso/talla ${ }^{2}$ ).

El perímetro braquial (PB)se midió con una cinta métrica, con graduación de hasta un milímetro. Se midió en el punto medio, calculado entre el acromion y cl olćcranon, con el brazo en extensión y relajado, sin que la cinta medidora deprimiera la piel del alumno.

El pliegue cutáneo tricipital (PCT), se midió tomando un pliegue en el punto medio de la distancia entre el acromion y el olécranon, en la cara posterior del brazo izquierdo, con un calibrador tipo HOLTAIN, con una precisión de $0,2 \mathrm{~mm}$. La medida se tomó tres veces en cada alumno, hallándose posteriormente el valor medio de las tres mediciones.

Todos los instrumentos de medida, se verificaban en cada sesión y se procedía a su calibración si era necesario.

La recogida de los datos antropométricos la llevó a cabo un solo observador (un médico), y se realizó en cada uno de los centros escolares, en una sala habilitada para la ocasión.
Para la codificación y análisis descriptivo de los datos se utilizó el programa informático EPIINFO versión 6.0, y las variables se expresaron como medias (DE), así como en porcentaje de sujetos respecto a los percentiles de referencia 3, 50 y 97. Como medidas de comparación se utilizaron las tablas y curvas de crecimiento de Hernández del Instituto de Investigación sobre el Crecimiento de la Fundación Orbegozo ${ }^{7}$.

Para las comparaciones entre las variable se utilizó la t de student, tomando como nivel de significación un valor de $\mathrm{p} \leq 0.05$.

La valoración del sobrepeso se realizó tomando como punto de corte un IMC $\geq 25$ $\mathrm{Kg} / \mathrm{m}^{2}$, según recomendación de diferentes autores ${ }^{8,9}$.

\section{RESULTADOS}

Del total de niños estudiados (493), 51,1\% eran varones $(48.9 \%$ niñas) con la distribución por edad y sexo de las variables estudiadas que se reflejan en las tablas 1 y 2 .

Las tallas medias, en todas las edades y sexos de los escolares, fueron superiores a las estándares de referencia, siendo esta diferencia mayor en los más pequeños para ir disminuyendo paulatinamente a medida que aumentaba la edad. Sin embargo, cabe destacar el descenso observado en este parámetro a la edad de 11 años en ambos sexos (figura 1).

En los varones, solamente un alumno $(0.4 \%)$, quedó por debajo del percentil 3 de las tablas de referencia y el $11.1 \%$ del total se encontraba por encima del P97 (tabla 3).

En las niñas, 3 alumnas (1.2\%) quedaron por debajo del P3 y el $7.4 \%$ por encima del P97 (tabla 3).

Los valores medios de peso para las distintas edades y sexos, fueron superiores a los estándares en casi todas las edades, siendo esta diferencia más evidente en los grupos 
Tabla 1

Parámetros antropométricos (Valores medios y desviaciones estándar) obtenidos en los escolares de la localidad de Ubrique, Cádiz

\begin{tabular}{|rccccccccccccc|}
\hline Edad & \multirow{2}{*}{${ }^{\circ}$ Alumnos } & \multicolumn{3}{c}{ Talla } & \multicolumn{3}{c}{ Peso } & \multicolumn{3}{c}{ Pliegue cutáneo } & \multicolumn{3}{c|}{ Perímetro braquial } \\
\hline & Niñas & Niños & Niñas & Niños & Niñas & Niños & Niñas & Niños & Niñas & Niños \\
\hline 4 & 15 & 14 & $106.9(4.9)$ & $105.4(4.7)$ & 18.2 & $(3.4)$ & 17.9 & $(2.5)$ & $10.2(1.6)$ & $10.8(3.9)$ & $17.2(2.5)$ & $17.3(1.3)$ \\
5 & 18 & 21 & $110.6(3.1)$ & $114.3(4.4)$ & 19.7 & $(2.0)$ & 20.7 & $(2.4)$ & $10.5(1.9)$ & $10.0(2.1)$ & $18.3(1.6)$ & $18.0(1.5)$ \\
6 & 22 & 23 & $116.3(3.8)$ & $119.6(4.1)$ & 21.7 & $(2.9)$ & 22.4 & $(4.1)$ & $10.6(3.0)$ & $9.8(5.2)$ & $18.6(2.3)$ & $18.2(2.1)$ \\
7 & 21 & 25 & $121.9(4.5)$ & $123.5(4.0)$ & 24.3 & $(3.7)$ & 25.6 & $(4.8)$ & $12.1(4.0)$ & $10.6(3.8)$ & $19.2(1.8)$ & $19.1(2.4)$ \\
8 & 22 & 21 & $128.2(4.5)$ & $129.8(4.5)$ & 27.9 & $(6.9)$ & 28.3 & $(4.7)$ & $13.8(7.1)$ & $11.9(5.1)$ & $20.2(3.1)$ & $19.4(2.8)$ \\
9 & 27 & 23 & $133.1(5.5)$ & $134.4(5.9)$ & 30.3 & $(4.6)$ & 30.7 & $(2.7)$ & $15.0(5.3)$ & $11.5(4.2)$ & $21.0(2.8)$ & $19.9(1.6)$ \\
10 & 24 & 32 & $142.0(6.0)$ & $140.2(6.4)$ & 36.0 & $(6.9)$ & 38.0 & $(9.5)$ & $15.2(3.9)$ & $15.2(8.0)$ & $21.3(2.2)$ & $22.1(3.5)$ \\
11 & 23 & 26 & $142.5(8.9)$ & $142.4(5.3)$ & 34.5 & $(8.9)$ & 35.4 & $(6.5)$ & $12.2(4.4)$ & $13.0(7.9)$ & $21.1(2.9)$ & $20.8(3.7)$ \\
12 & 29 & 26 & $151.5(6.5)$ & $148.7(7.2)$ & 44.2 & $(9.1)$ & 42.7 & $(8.0)$ & $16.1(5.1)$ & $12.9(4.7)$ & $23.7(3.0)$ & $22.6(2.4)$ \\
13 & 28 & 28 & $155.8(7.0)$ & $156.6(7.7)$ & $51.1(13.1)$ & 48.2 & $(8.2)$ & $18.9(7.7)$ & $12.5(6.7)$ & $24.9(3.5)$ & $23.6(2.5)$ \\
14 & 12 & 13 & $156.2(5.0)$ & $162.2(8.8)$ & 51.2 & $(6.6)$ & $57.1(13.5)$ & $17.0(5.4)$ & $13.6(9.3)$ & $25.1(2.5)$ & $25.8(4.3)$ \\
\hline
\end{tabular}

Tabla 2

Índice de masa corporal. Valores medios y desviaciones estándar en los escolares de Ubrique y población de referencia

\begin{tabular}{|c|c|c|c|c|c|c|}
\hline \multirow{3}{*}{ Edad } & & & \multicolumn{4}{|c|}{ Indice de masa corporal } \\
\hline & \multicolumn{2}{|c|}{ N."Alumnos } & \multicolumn{2}{|c|}{ Niñas } & \multicolumn{2}{|c|}{ Niños } \\
\hline & Niñas & Niños & Ubrique & Estándar & Ubrique & Estándar \\
\hline 4 & 15 & 14 & $15.9(2.3)$ & $16.2(1.5)$ & $16.1(1.9)$ & $16.2(1.2)$ \\
\hline 5 & 18 & 21 & $16.1(1.4)$ & $16.2(1.6)$ & $15.8(1.5)$ & $16.0(1.2)$ \\
\hline 6 & 22 & 23 & $16.0(1.7)$ & $16.5(1.8)$ & $15.6(2.1)$ & $16.3(1.4)$ \\
\hline 7 & 21 & 25 & $16.3(1.9)$ & $16.6(2.1)$ & $16.7(2.7)$ & $16.5(1.7)$ \\
\hline 8 & 22 & 21 & $16.8(3.2)$ & $16.8(2.1)$ & $16.8(2.5)$ & $16.7(1.8)$ \\
\hline 9 & 27 & 23 & $17.0(2.3)$ & $17.1(2.3)$ & $17.0(1.3)$ & $16.8(2.0)$ \\
\hline 10 & 24 & 32 & $17.8(2.7)$ & $17.5(2.4)$ & $19.1(3.6)^{* *}$ & $17.1(2.1)$ \\
\hline 11 & 23 & 26 & $16.8(3.0)^{*}$ & $18.2(2.6)$ & $17.4(2.6)$ & $17.7(2.4)$ \\
\hline 12 & 29 & 26 & $19.1(3.0)$ & $18.6(2.6)$ & $19.2(2.7)$ & $18.2(2.5)$ \\
\hline 13 & 28 & 28 & $20.8(3.7)^{*}$ & $19.3(2.8)$ & $19.5(2.5)$ & $18.7(2.6)$ \\
\hline 14 & 12 & 13 & $20.9(2.3)$ & $20.4(2.8)$ & $21.7(4.9)^{*}$ & $19.4(2.8)$ \\
\hline
\end{tabular}

$* p<0.05 ; * * p<0,01$.

de edad mayor. Al igual que en la talla, volvió a observarse una bajada a los 11 años en ambos sexos (figura 1).

Hay que destacar que 5 alumnos varones $(1.9 \%)$ y 4 niñas $(1.6 \%)$ se situaron por debajo del P3 y $10.7 \%$ de alumnos y $6.2 \%$ de alumnas por encima del P97. (tabla 3).

En el perímetro braquial como en los parámetros anteriores, los escolares estudiados presentaban valores medios superiores a los considerados estándares, salvo a los 11 en las niñas (figura 2).

En la comparación de esta variable con los percentiles de referencia, sólo una alumna (0.4\%) presentaba cifras por debajo del P3 y el $9.5 \%$ de ellas, se situaban por encima del P97 (tabla 3).

En los varones, no encontramos ningún alumno por debajo del P3 y el $10.7 \%$, por encima del P97 (tabla 3). 
Figura 1

Talla y peso de los escolares de Ubrique y población de referencia
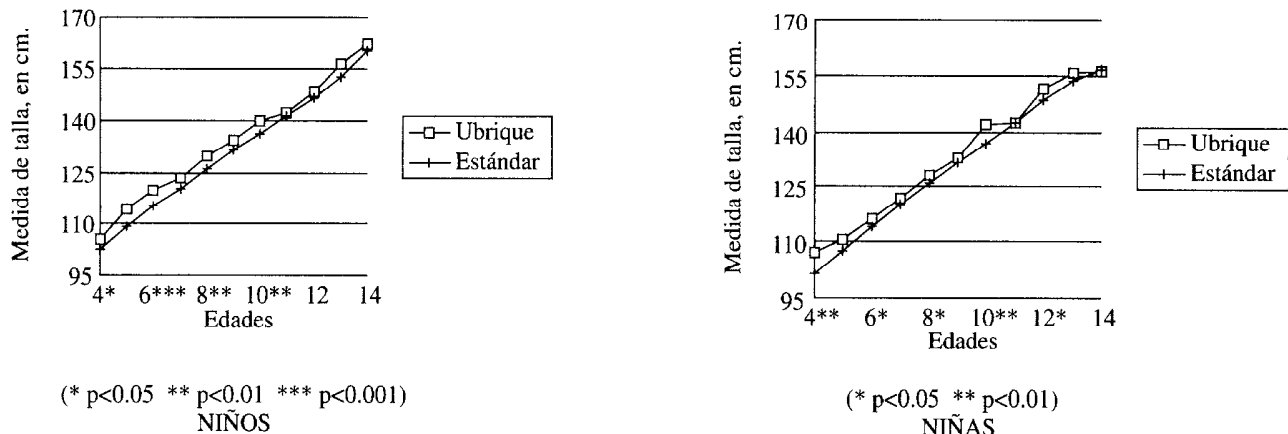

$\left({ }^{*} \mathrm{p}<0.05 * * \mathrm{p}<0.01\right)$

NIÑAS
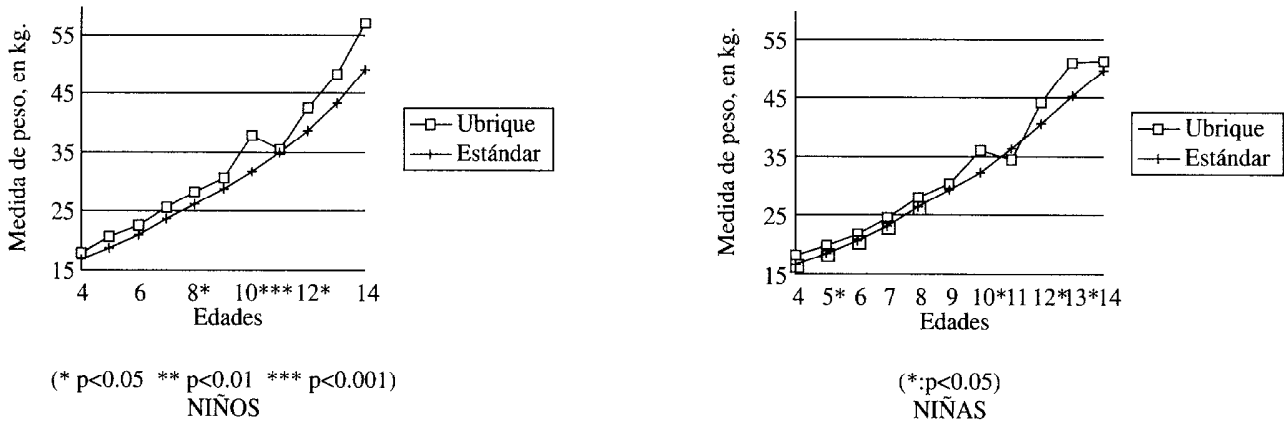

- - - Ubrique
+ - Estándar Edades

$(*: \mathrm{p}<0.05)$

NIÑAS

Figura 2

Perímetro braquial y pliegue cutáneo tricipital de los escolares de Ubrique y población de referencia

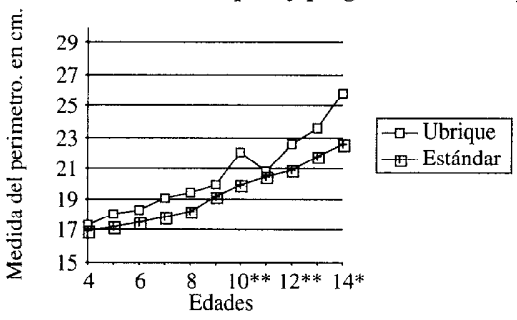

$\left(* \mathrm{p}<0.05^{* *} \mathrm{p}<0.01{ }^{* * *} \mathrm{p}<0.001\right)$ NIÑOS
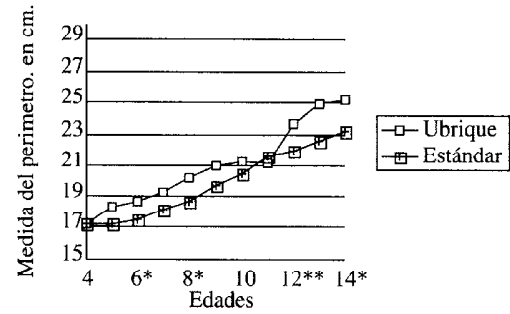

$(* \mathrm{p}<0.05 * * \mathrm{p}<0.01)$ NIÑAS
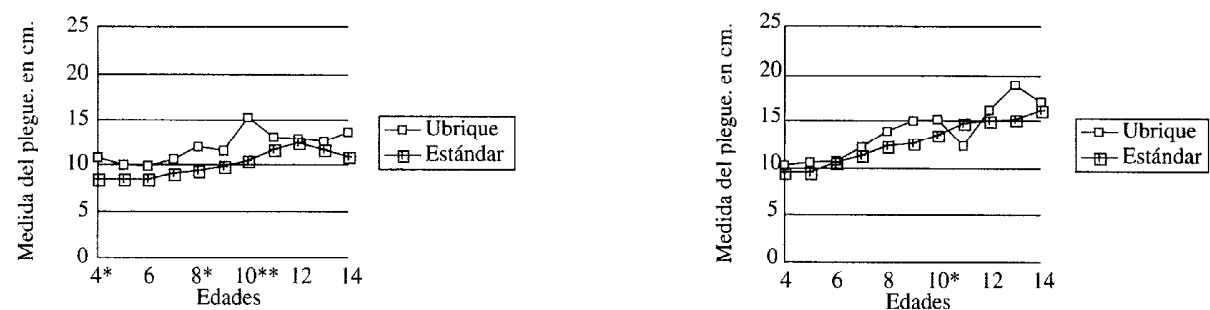

$\left({ }^{*} \mathrm{p}<0.05 * * \mathrm{p}<0.01\right)$ NIÑOS

NIÑAS 
Los valores del pliegue cutáneo tricipital, volvieron a ser superiores a los de Hernández et al. ${ }^{7}$ en todas las edades, salvo a los 11 en las niñas (figura 2).

En la valoración percentilada del pliegue cutáneo de la población estudiada, nos encontramos que el $6.9 \%$ de cstos alumnos, en su mayoría varones $(10.3 \%)$, se situaban en valores extremos de este parámetro (> P97) (tabla 3 ).

Finalmente y respecto al IMC, no se encontraron diferencias significativas entre nuestra población y la de Hernández et al. ${ }^{7}$, salvo en los varones de 10 y 14 años que fucron superiores al estándar, y las niñas de 11 que presentaban valores más bajos, y las de 13 años con valores algo superiores. En general puede apreciarse, que los valores en la población estándar eran superiores en las edades más jóvenes ( 4 a 7 años), mientras que en los niños de Ubrique de ambos sexos los resultados eran superiores en las edades mayores (12 a 14 años) (tabla 2).
Si consideramos al porcentaje de niños con valor del IMC superior al P97, observamos que existen $9.5 \%$ de varones y $7.1 \%$ de niñas que se situaban a este nivel. Igualmente si consideramos en sobrepeso a aquellos alumnos con IMC $>25 \mathrm{Kg} / \mathrm{m}^{2}$, vemos que solo 20 niños (4\%) se encontraban en esta situación, con un mayor número de casos a partir de los 12 años (13 casos), sin que existieran grandes diferencias por sexos (55\% varones y $45 \%$ niñas).

\section{DISCUSIÓN}

Diferentes autores, coinciden al señalar que el nivel de salud y nutrición de una comunidad infantil, se debe evaluar con parámetros referidos al crecimiento y desarrollo. La técnica antropométrica ofrece un método práctico y sencillo para esta valoración a nivel poblacional ${ }^{10}$. Sin embargo, la validez de sus procedimientos ha sido a veces cuestionada ya que va a depender de una serie de factores como la elección de parámetros su-

Tabla 3

Distribución de las medidas antropométricas obtenidas en los escolares de la localidad de Ubrique respecto a la población de referencia

\begin{tabular}{|c|c|c|c|}
\hline Medidas y Percentiles & Niñas $\%(n)$ & Niños $\%(n)$ & Total \% \\
\hline \multicolumn{4}{|l|}{ Talla } \\
\hline$\leq \mathrm{P} 3$ & $1.24(3)$ & $0.39(1)$ & 0.81 \\
\hline$>$ P50 & $65.14(157)$ & $76.98(194)$ & 71.19 \\
\hline$>\mathrm{P} 97$ & $7.46(18)$ & $11.11(28)$ & 9.33 \\
\hline \multicolumn{4}{|l|}{ Peso } \\
\hline$\leq \mathrm{P} 3$ & $1.65(4)$ & $1,98(5)$ & 1.82 \\
\hline$>$ P50 & $58.09(140)$ & $65.07(164)$ & 61.66 \\
\hline$>\mathrm{P} 97$ & $6.22(15)$ & $10.71(27)$ & 8.51 \\
\hline \multicolumn{4}{|l|}{ P. Braquial } \\
\hline$\leq \mathrm{P} 3$ & $0.41(1)$ & $0.00(0)$ & 0.20 \\
\hline$>$ P50 & $65.14(157)$ & $69.84(176)$ & 67.54 \\
\hline$>\mathrm{P} 97$ & $9.54(23)$ & $10.71(27)$ & 10.14 \\
\hline \multicolumn{4}{|l|}{ P. Cutáneo } \\
\hline$\leq \mathrm{P} 3$ & $0.82(2)$ & $0.79(2)$ & 0.81 \\
\hline$>$ P50 & $54.35(131)$ & $54.36(137)$ & 54.36 \\
\hline$>$ P97 & $3.31(8)$ & $10.31(26)$ & 6.89 \\
\hline \multicolumn{4}{|l|}{ I. M. C. } \\
\hline$\leq \mathrm{P} 3$ & $1.65(4)$ & $1.98(5)$ & 1.82 \\
\hline$>$ P50 & $43.56(105)$ & $50.39(127)$ & 47.05 \\
\hline$>P 97$ & $7.05(17)$ & $9.52(24)$ & 8.31 \\
\hline
\end{tabular}


ficientemente sensibles, la precisión en la recogida de datos, la utilización de estándares o patrones de referencia adecuados y la interpretación correcta de los resultados.

En este trabajo se pretende conocer el perfil antropométrico y la prevalencia de sobrepeso de un colectivo de alumnos ubicados en una zona de la sierra gaditana que a pesar de tener carácter rural, posee una marcada actividad industrial, con una bolsa importante de economía sumergida que dificulta el conocimiento del nivel socioeconómico real de la población ${ }^{11}$.

Con relación a la metodología utilizada, hay que decir que la recogida de la información por un solo observador y con medidas repetidas, avala la fiabilidad del estudio. Igualmente el haber sido realizado en una muestra importante de los escolares de la zona, nos permite considerar los resultados como representativos de la población en este grupo de edad. Por otra parte, los parámetros antropométricos utilizados son los más generalmenteaceptados ${ }^{12}$ y se ajustan bastante a lo establecido en el Consenso Español de $1995^{\circ}$.

Con respecto al estándar utilizado, elegimos el de Hernández et al. ${ }^{7}$ pues a pesar de ser un trabajo realizado en una población de una zona geográficamente bastante diferente, se trata de un estudio longitudinal muy utilizado como referencia en pediatría.

Entre los resultados obtenidos, se podría destacar que los niños de Ubrique presentan en los parámetros estudiados valores superiores al estándar de referencia, a excepción de los 11 años de edad en ambos sexos, donde los valores fueron siempre inferiores. Este hecho no parece debido a un problema metodológico ya que no se observa en otros trabajos que utilizan la misma metodología ${ }^{13}$, y podría ir a favor de un efecto cohorte susceptible de ser comprobado en estudios posteriores.

Nuestros resultados fueron, igualmente superiores a los encontrados por Moreno et al. ${ }^{14}$ y Goñi et al. ${ }^{15}$ en la comunidad de Madrid. Esto iría a favor de la hipótesis de que los cambios geográficos y climáticos, podrían explicar las diferencias entre los autores, y podría ser corroborado por otros trabajos desarrollados en otras comunidades autónomas. Sin embargo, en un trabajo realizado en Murcia ${ }^{16}$ todos los parámetros estudiados presentaron valores superiores a los nuestros, preferentemente en edades mayores, a excepción del PCT que fue superior en nuestros escolares varones. En este sentido, también hay que destacar el estudio realizado por Doña et al. en Málaga ${ }^{17,18}$, donde a pesar de la proximidad geográfica y climática, se encontraron valores superiores a los nuestros en todos los parámetros. Parece por tanto, lógico pensar que las diferencias entre los distintos estudios, no deberían ser atribuidas únicamente a factores ambientales. Aunque en contra de esto, vemos que en el trabajo realizado por Carvajal et al. ${ }^{13}$ en Chiclana de la Frontera (Cádiz), se obtuvieron resultados bastante similares a los de Ubrique, y en el de Ruiz Jiménez et al. ${ }^{19}$ realizado en Cádiz, tampoco se observaron diferencias significativas con nuestro grupo en el PCT y IMC.

Otro factor importante que podría condicionar las diferencias encontradas es el nivel socioeconómico. Sandín ${ }^{20}$ y otros autores ${ }^{21}$, afirman que en nuestro país existen claras diferencias en el proceso de crecimiento y desarrollo entre distintas regiones, asociados fundamentalmente (aunque no únicamente) a sus distintas caractcrísticas socioeconómicas. Nosotros no pudimos comprobar su efecto sobre nuestros escolares debido a la dificultad para obtener unos indicadores fiables del nivel socioeconómico de la población, a causa de su particular economía.

Respecto a la valoración del sobrepeso, destaca el bajo porcentaje de escolares con IMC por encima del P97 de referencia. La elección del P97 como punto de corte en lugar del P90 ${ }^{8,22}$ o incluso del P85 $5^{5}$ utilizado por otros autores, ha estado condicionado por el uso de las tablas de Hernández et al. que se tomaron como medida de comparación. Esta utilización del P97 en el IMC nos llevó a identificar solo sujetos con un sobrepeso elevado, datos que corresponden más a obesidad que a sobrepeso, con la consiguien- 
te subestimación de la prevalencia en nuestra población. Sin embargo, a favor de la baja prevalencia de sobrepeso está el que también se observó un pequeño porcentaje de casos cuando se utilizó como medida de referencia el $\mathrm{IMC} \geq 25 \mathrm{Kg} / \mathrm{m}^{2}$.

Estos resultados parecen estar en línea con lo obtenido en el trabajo sobre valoración nutricional de estos escolares recientemente publicado ${ }^{6}$, donde se concluía que su alimentación se ajustaba bastante a las Recomendaciones Dietéticas de energía y nutrientes aunque con un perfil más occidental que mediterráneo.

\section{BIBLIOGRAFÍA}

1. Penchaszadech VB. Condicionantes básicos para el crecimiento. Una larga polémica: herencia o ambiente. En: OPS. Crecimiento y desarrollo: hechos y tendencias. Publ Cientif n. ${ }^{\circ}$. Washington 1988: 90-101.

2. Wolfe WS, Campbell CC, Frongillo EA, Haas JD, Melnik TA. Overweight schoolchildren in New York State: Prevalence and characteristics. Am J Public Health 1994; 84: 807-813.

3. Serdula MK, Ivery D, Coates RJ, Freedman DS, Williamson DF, Byers T. Do obese children become obese adults? A Review of the literatura. Prev Med 1993; 22: 167-177.

4. Rolland-Cachera M, Deheer M, Guilloud-Bataille M. Tracking the development of adiposity from one month of age to adulthood. Ann Hum Biol 1987; $14: 219-229$.

5. Albert B, DiGiuseppi C. Guide to clinical preventive services: screening for obesity. En: U.S. task force guide to clinical preventive service, 2 nd edition. (Internet). 1996

6. Failde I, Zafra JA, Ruiz E, Novalbos JP. Valoración de la alimentación de los escolares de una población de la Sierra de Cádiz (Ubrique). Med Clin (Barc) 1997; 198: 254-258.

7. Hernández M, Catallet J, Narvaiza JL, Rincón JM, Ruiz I, Sanchez E. et al. Curvas y tablas de crecimiento. Bilbao: Fundación F. Orbegozo, Instituto de Investigación sobre Crecimiento y Desarrollo; 1988

8. Sociedad Española para el Estudio de la Obesidad (SEEDO). Consenso español 1995 para la evaluación de la obesidad y para la realización de estudios epidemiológicos. Med Clin (Barc) 1996; 107: 782-787.
9. Serra Majem LL. Sobrepeso y obesidad como problema de salud pública. En: Nutrición y Salud Pública: Métodos, bases científicas y aplicaciones. Lluis Serra Majem, Javier Aranceta Bartriha, José Mataix Verdú eds., Barcelona: Masson; 1995. p. 237-243.

10. Alastrué V. Antropometría y obesidad. Med Clin(Barc) 1994; 102: 16-19.

11. Ruiz E. Estudio de alimentación y de salud bucodental en una población escolar gaditana: Ubrique. [T'esis Doctoral ]. Cádiz: Facultad de Medicina, Universidad de Cádiz; 1992.

12. Alastrué A, Rull M, Camps I, Ginesta C, Melus MR, Salvá JA. Nucvas normas y consejos en la valoración de los parámetros antropométricos en nuestra población: índice adiposo muscular, índices ponderales y tablas de percentiles de los datos antropométricos útiles en una valoración nutricional. Med. Clin. (Barc) 1988; 91: 223-236.

13. Carvajal N, Alcaraz M, Alcaraz E, Failde I. Valoración del estado nutricional de una población de escolares gaditanos. Rev San Hig Púb 1993; 67: 359-367.

14. Moreno B, Monereo S, Moreno FJ, Desco M. Curvas de crecimiento de los niños de la Comunidad Autónoma de Madrid. Salud Rural 1987; 2: 41-49.

15. Goñi I, Bayón C, García-Diz L. Estudio antropométrico y estado nutritivo de una población escolar del cinturón industrial de Madrid. Rev Esp Pediatr $1985 ; 41291-296$.

16. Hernández AM, Tébar FJ, Serrano S, Alvarez Y, Illán F, Valdés M. Estudio antropométrico de la población escolar de la Comunidad Autónoma de Murcia. Med Clín (Barc) 1992; 98: 651-655.

17. Doña JL, Bueno A, Martínez A. Valoración de los parámetros antropométricos nutricionales: perímetro braquial y pliegues cutáneos en escolares de Malaga ciudad. An Esp Pediatr 1990; 33: 225-228.

18. Doña JL, Bueno A, Martínez A. Estudio de los parámetros antropométricos peso, talla y perímetro cefálico en escolares de Málaga ciudad. An Esp Pediatr 1990; 33: 229-232.

19. Ruiz MA, Martínez JM, Cano MC. Valoración antropométrica del estado nutricional de una población adolescente de Cádiz. Enf Científica 1996; 174: 17-23.

21. Goñi Y, García L. Influencia del nivel socioeconómico en el crecimiento infantil. Rev Nutr Clin 1988; 4: 42-47

22. Rey Calero del J, Gil Miguel A, Calle Purón ME, Lasheras Lozano ML, Alegre del Rey E. Estudio epidemiológico del índice de masa corporal en una población escolar de Madrid. Rev San Hig Pub 1992; 66: 65-70. 